\title{
GMRT detection of a new wide-angle tail (WAT) radio source associated with the galaxy PGC 1519010
}

\author{
N. G. Kantharia ${ }^{1}$, M. Das ${ }^{2}$, Gopal-Krishna ${ }^{1}$ \\ ${ }^{1}$ National Centre for Radio Astrophysics, Tata Institute of Fundamental Research, \\ Post Bag 3, Ganeshkhind, Pune University Campus, Pune-411007, India \\ ${ }^{2}$ Raman Research Institute, Sadashivnagar, Bangalore-560080, India
}

2018 October 30

\begin{abstract}
.
We report the serendipitous detection of a Wide-Angle-Tail (WAT) radio galaxy at 240 and $610 \mathrm{MHz}$, using the Giant Metrewave Radio Telescope (GMRT). This WAT is hosted by a cD galaxy PGC 1519010 whose photometric redshift given in the SDSS DR6 catalog is close to the spectroscopic redshifts $(0.105,0.106$ and 0.107$)$ of three galaxies found within $4^{\prime}$ of the $\mathrm{cD}$. Using the SDSS DR6 we have identified a total of 37 galaxies within $15^{\prime}$ of the $\mathrm{cD}$, whose photometric redshifts are between 0.08 and 0.14 . This strongly suggests that the $\mathrm{cD}$ is associated with a group of galaxies whose conspicuous feature is a north-south chain of galaxies (filament) extending to at least $2.6 \mathrm{Mpc}$. The ROSAT All-Sky Survey shows a faint, diffuse X-ray source in this direction, which probably marks the hot intra-cluster gas in the potential well of this group.

We combine the radio structural information for this WAT with the galaxy clustering in that region to check its overall consistency with the models of WAT formation. The bending of the jet before and after its disruption forming the radio plume, are found to be correlated in this WAT, as seen from the contrasting morphological patterns on the two sides of the core. Probable constraints imposed by this on the models of WAT formation are pointed out. We also briefly report on the other interesting radio sources found in the proximity of the WAT. These include a highly asymmetric double radio source and an ultra-steep spectrum radio source for which no optical counterpart is detected in the SDSS.
\end{abstract}

Key words: Radio Galaxies: Cluster of galaxies: Ram pressure: Intracluster medium 


\section{Introduction}

Wide-Angle Tail (WAT) are a subset of radio galaxies near the Fanaroff-Riley (1974) luminosity transition, which have been extensively discussed because of their exclusive association with cluster dominant (cD) galaxies and also because of the abrupt flaring of their jets after maintaining a well collimated flow to distances $\geq 20 \mathrm{kpc}$ from the core (e.g. Owen\& Rudnick 1976, O'Donoghue et al. 1993) The jet disruption is sudden unlike FR I jets and the resulting plumes are often sharply bent. On the other side, although the jets are collimated like FR II galaxies, they do not terminate in hot spots. Search for explanations of the WAT phenomenon began in the 1980s (e.g. Burns 1981; Eilek et al. 1984; Leahy 1984; O'Dea \& Owen 1985; O'Donoghue et al. 1990). Since neither the bending of the radio plumes occurs universally, nor is the bending of jet essential for its flaring and plume formation, jet bending is currently regarded as a phenomenon independent from jet flaring (Hardcastle \& Sakelliou 2004; Hardcastle, Sakelliou \& Worrall 2005).

Since the host galaxies of WATs are the dominant members of their group/cluster and hence located close to the bottom of the gravitational potential well, they are not expected to have a large motion relative to the intra-cluster medium (ICM). This situation is not conducive to large ram pressure that could bend the jet/plume leading to the WAT morphology. An alternative mechanism, based on numerical simulations has been investigated by Loken et al.(1995), Hooda \& Wiita (1996) and Burns et al. (1994). In this picture, the sudden disruption of the jet and bending of its plume can arise, if upon crossing a sharp transition between the interstellar medium (ISM) of its host galaxy and the ICM, the jet encounters a transonic crosswind of the ICM resulting from cluster merger (an analytical treatment of the jet propagation through an ISM/ICM interface can be found in Gopal-Krishna \& Wiita 1987). Another alternate mechanism proposed in Loken et al. (1995) invokes a jet crossing an oblique shock formed due to colliding clumps in the cluster. Strong support for the merging cluster scenario comes from the detection of X-ray elongations which trace the merger axis and are found to be in the direction of the WAT radio tails (Gomez et al. 1997) and perpendicular to the initial jet direction (Burns et al. 1994). Another mechanism, by Higgins, O'Brian \& Dunlop (1999), associates the jet flaring with its encounter with a discrete clump in the ICM. While all these mechanisms seem plausible, it is intriguing that the deep Chandra imaging, which is available for the best known WAT, namely 3C 465 (Hardcastle et al. 2005), has failed to reveal any discontinuity in the external medium at the locations where the jets flare. Moreover, for this same WAT, Jetha et al. (2006) \& Hardcastle et al. (2005) have argued that, if the jet/plume is extremely light relative to the external medium, the speed of the host galaxy required for jet bending is only $\sim 100$ $\mathrm{kms}^{-1}$, which is not implausibly high even for the central galaxies of rich clusters (much higher speeds can occur for galaxies in merging clusters). Thus, the bending of the jet/plume in WATs can possibly be explained.

The circumstance of jet termination in WATs has been investigated in several studies. Hardcastle \& Sakelliou (2004) have shown that the distance between the 
host galaxy and the base of the plume inversely correlate with the cluster richness as quantified in terms of the temperature of the ICM (also, Jetha et al. 2006). Since WATs reside at or close to gravitational centres of clusters and groups of galaxies, these can also be used as a signpost for the cluster or group of galaxies. Indeed, Blanton et al. (2000, 2001, 2003) have identified several clusters using the WAT sources detected in the FIRST survey (Becker et al. 1995).

In this paper, we report the GMRT detection of a WAT associated with the galaxy PGC 1519010 (SDSS J113920.37+165206). The radio source was noticed by us while imaging the radio continuum of the low surface brightness galaxy UGC 6614. This was interesting, given that the galaxy PGC 1519010 has itself been catalogued as a low surface brightness galaxy (U1-3) by O'Neil et al. (1997). In this paper, we discuss the nature of this radio source and its optical/X-ray environment. A Hubble constant of $70 \mathrm{kms}^{-1} \mathrm{Mpc}^{-1}$ is assumed.

\section{PGC 1519010 - the host galaxy}

PGC 1519010 is located $\sim 16^{\prime}$ to the south of UGC 6614, the giant low surface brightness (LSB) galaxy that was the principal target of our observations as part of our larger programme of observing the radio-band AGN activity in giant low surface brightness galaxies (see Das et al. 2007, 2008). We noticed that PGC 1519010 is catalogued by O'Neil et al. (1997) as the low surface brightness galaxy U1-3. The catalogue contains all those galaxies in the region of the Cancer and Pegasus clusters whose central surface brightness $\mu(0) \geq 22.0$ mag- $\operatorname{arcsec}^{-2}$ (O'Neil et al. 1997). Curiously, O'Neil et al. found that U1-3 has a central surface brightness of $\mu_{b}(0)=22.39$ mag-arcsec ${ }^{-2}$ and that its radial brightness distribution is better fit by the King's model $(1962,1966)$, rather than the exponential profile characteristic of LSB (disk) galaxies. Since King's model is known to describe the surface brightness distribution of globular clusters in our galaxy, O'Neil et al. had in fact suggested that U1-3 (PGC 1519010) might be a LSB globular cluster. A closer inspection of the SDSS image, however, showed that it is an elliptical galaxy, most probably a $\mathrm{cD}$ with a large halo extending in the north-south (Fig. 1). Such a corehalo distribution of stars can be explained in terms of tidal distortion of an elliptical (with a typical de Vaucouleurs $\mathrm{r}^{1 / 4}$ brightness profile) by its repeated gravitational encounters with other galaxies (e.g., Kormendy 1997), a highly plausible scenario for central regions of clusters and groups of galaxies.

Thus, we point out that the galaxy PGC 1519010 classified by O'Neil et al. (1997) as an LSB galaxy needs to be reclassified as an elliptical in view of its corehalo stellar distribution (see Fig. 1), akin to $\mathrm{cD}$ galaxies generally found near the centres of clusters/groups of galaxies. The main properties of this galaxy obtained from literature are summarized in Table. 1.

Figs. $2 \mathrm{a} \& \mathrm{~b}$ shows the radio continuum images of the WAT radio galaxy at 21 $\mathrm{cm}$, reproduced from the FIRST survey (Becker et al. 1995) and the NVSS survey (Condon et al. 1998). The twin-lobed morphology consists of a diffuse radio lobe 


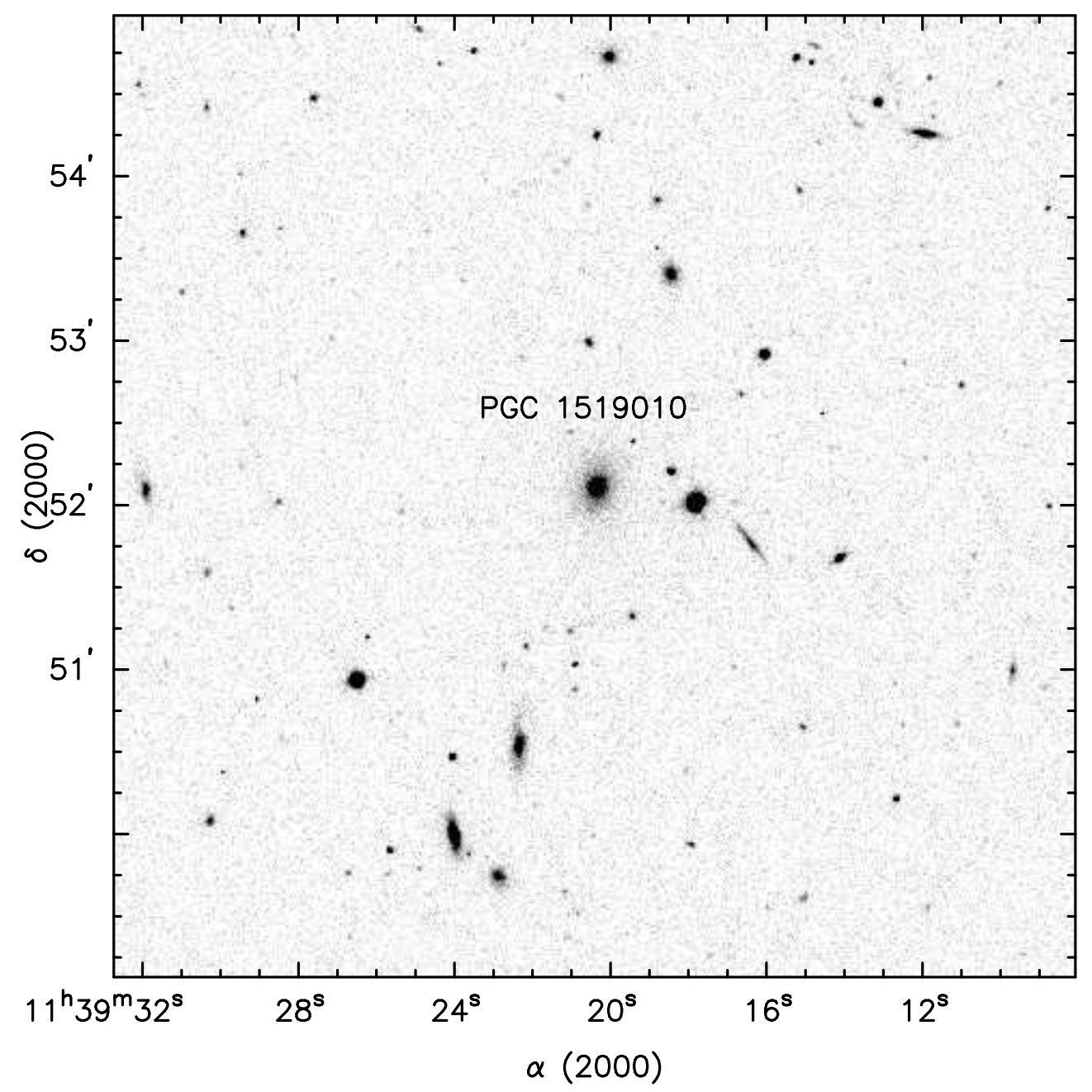

Figure 1. PGC 1519010 (U1-3) in optical - from the SDSS. Notice the core-halo type of morphology indicating that it is a cD type elliptical with a large stellar halo extended north-south.

west of the core and a diffuse eastern lobe which suggests that the eastern jet has undergone bending prior to flaring. Such a morphology is indicative of WATs and we report here its structural details based on our GMRT observations. We also discuss the optical field around this galaxy, as well as the faint halo of soft X-rays picked up in the ROSAT all sky survey (Voges et al. 1999 and references therein).

\section{GMRT observations and results}

The field was observed on 30 December 2005 using GMRT (Swarup et al. 1991; Ananthakrishnan \& Rao 2002), in the dual-frequency mode which allows simulta- 


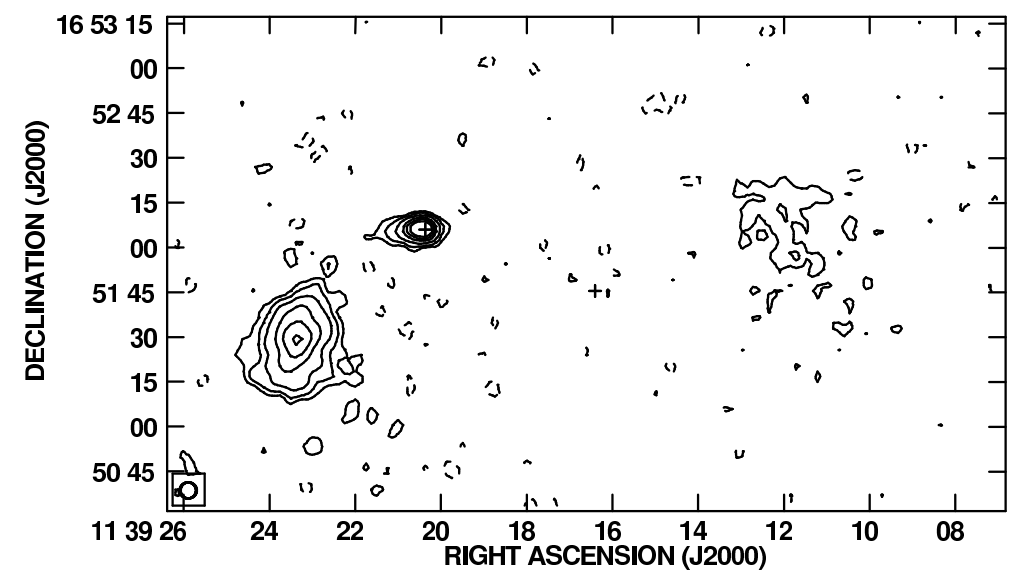

Figure 2. (a) PGC 1519010 in $21 \mathrm{~cm}$ radio continuum from the FIRST survey (resolution $\left.5.7^{\prime \prime}\right)$. Notice the double-lobed structure around the core of the galaxy. Notice the jets emerging from the central galaxy in the FIRST map. The first contour is $0.36 \mathrm{mJy} / \mathrm{beam}$ and thereafter the contours are plotted in multiples of 2. Notice the bent radio morphology on the eastern side of the core.

neous observations at 240 and $610 \mathrm{MHz}$ (for details of the observations, see Das et al. 2008, in preparation). The data obtained in the native $l t a$ format were imported to and analysed using NRAO AIPS ${ }^{1}$. Data of single RFI-free channels were first gain-calibrated. Bandpass calibration was then applied, after which several frequency channels were collapsed to generate a continuum database. The $240 \mathrm{MHz}$ data were severely affected by radio frequency interference with the net result that only $1.2 \mathrm{MHz}$ of the total $6 \mathrm{MHz}$ was usable and the rest of the data had to be discarded. The $610 \mathrm{MHz}$ images were generated using a total bandwidth of 12.5 MHz. We made images employing the robust weighting scheme (Briggs 1995), setting robust $=0$ (between uniform and natural weighting) and robust $=5$ (natural weighting) at both frequencies. All the data were self-calibrated and primary beam corrected. The GMRT images are shown in Figs. $3 \mathrm{a} \& \mathrm{~b}$. We note that the robust $=0$ image at $240 \mathrm{MHz}$ has a highly elliptical beam whereas the robust=5 image at 610 $\mathrm{MHz}$ does not add any more information to the WAT structure and hence these are not presented here. Since the WAT is located about 16' south of the phase centre of our observations, the sensitivity is compromised, especially at $610 \mathrm{MHz}$ where the half power width of the primary beam is about 50 '.

A striking feature of this WAT, evident from all the maps is that the overall bending of the radio structure is conspicuous only on the eastern side of the core (see Figs. 2, 3). More clarity about the morphology emerges from a joint inspection of the GMRT $(610 \mathrm{MHz})$ and the FIRST $(1.4 \mathrm{GHz})$ maps, both having a resolution

\footnotetext{
${ }^{1}$ The National Radio Astronomy Observatory is a facility of the National Science Foundation operated under cooperative agreement by Associated Universities, Inc.
} 


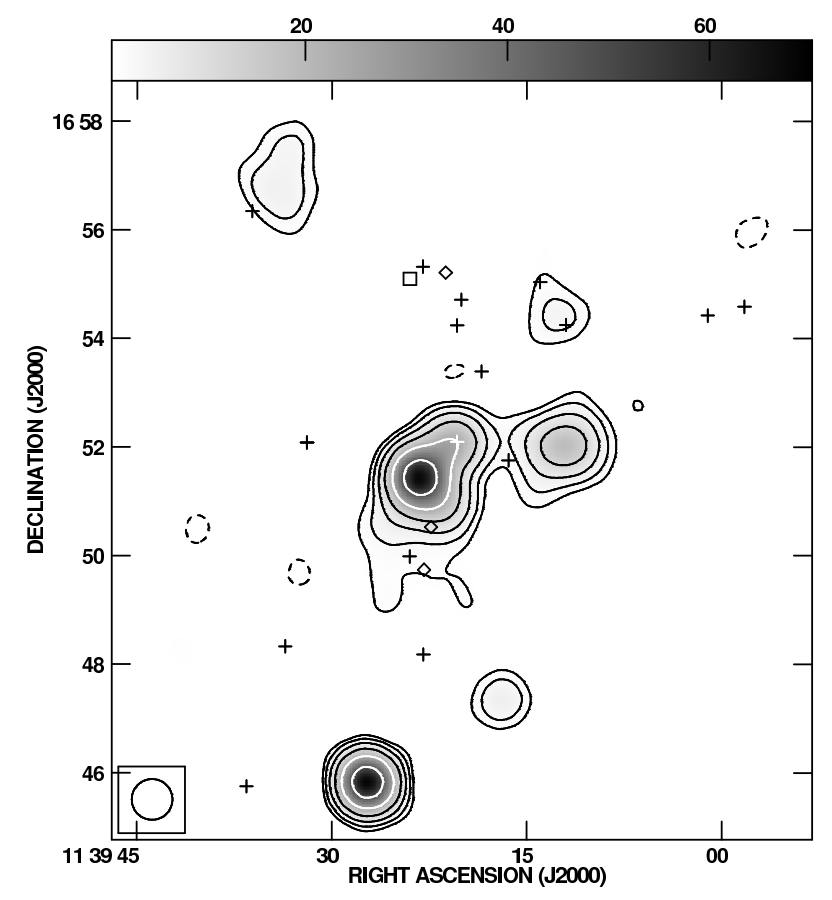

Figure 2. (b) PGC 1519010 in $21 \mathrm{~cm}$ radio continuum from the NVSS (resolution $45^{\prime \prime}$ ). Notice the double-lobed structure around the core of the galaxy. The first contour in the map is $1.5 \mathrm{mJy} /$ beam and thereafter the contours are plotted in multiples of 2 . Notice the bent morphology of the radio source. The plus (+) signs indicate the possible member galaxies of the group/cluster for which photometric redshifts are available, the diamonds indicate member galaxies for which spectroscopic redshifts are available and the square indicates the centre of the cluster given by Gal et al. (2003) which we discuss later in the paper.

of $\sim 6^{\prime \prime}$ (Figs. 2a \& 3a). It is seen that the twin jets emerge from the core along the east-west direction and form their lobes. Evidently, only the brighter eastern jet undergoes bending. This occurs gradually along the jet and the plume followed by a sharp bending (towards a position angle of about $220^{\circ}$ ) of the tail (see Fig. 3a). In stark contrast, no sign of a bent morphology is evident on the western side, despite the sudden flaring of the jet after propagating for nearly $230 \mathrm{kpc}$ from the core.

In Fig. 5 we show the spectral index distributions derived by combining the GMRT images with the NVSS and FIRST images at $1.4 \mathrm{GHz}$ (Fig. 2). For this, the GMRT $240 \mathrm{MHz}$ map was first smoothed to the 45" circular beam of the NVSS image. To examine the spectral index in the core region, the spectral index map was generated by combining the GMRT $610 \mathrm{MHz}$ and the $1.4 \mathrm{GHz}$ FIRST maps, after convolving the FIRST image to match the resolution of the $610 \mathrm{MHz}$ GMRT image $\left(7^{\prime \prime} \times 6^{\prime \prime}\right)$. The spectral index distributions were generated using the $>3 \sigma$ emission in the relevant maps. The spectrum is flat near the core $(\alpha=-0.4)$ and 
Table 1. Optical properties of PGC 1519010 from literature

\begin{tabular}{|c|c|c|}
\hline Parameter & Value & Reference \\
\hline \multirow[t]{3}{*}{ Names } & SDSS J113920.37+165206 & 1 \\
\hline & PGC 1519010 & 2 \\
\hline & 2MASX J11392034+1652058 & 3 \\
\hline Photmetric z1 & 0.138 & 1 \\
\hline Photmetric zCC2 & 0.112 & 1 \\
\hline Photmetric zD1 & 0.104 & 1 \\
\hline Radial brightness & Kings's model & 4 \\
\hline Morphological type code & $2.1 \pm 5$ & 2 \\
\hline Total apparent corrected B-mag & 16.69 & 2 \\
\hline Total apparent corrected I-mag & 15.1 & 2 \\
\hline Inclination & $35^{\circ}$ & 2 \\
\hline u-magnitude & 18.72 & 1 \\
\hline g-magnitude & 16.64 & 1 \\
\hline r-magnitude & 15.62 & 1 \\
\hline i-magnitude & 15.14 & 1 \\
\hline z-magnitude & 14.83 & 1 \\
\hline$\mu_{b}(0) \mathrm{mag} \operatorname{arcsec}^{-1}$ & 22.39 & 4 \\
\hline Inclination angle & $47.9^{\circ}$ & 4 \\
\hline Major axis upto $\mu_{b}=25$ isophote & $17.9^{\prime \prime}$ & 4 \\
\hline Closeby cluster & NSC $113924+165506$ & 5 \\
\hline
\end{tabular}

1. SDSS DR6

2. Hyperleda, Paturel et al. (2003)

3. NED

4. O'Neil et al. (1997)

5. Gal et al. (2003)

starts steepening along the eastern jet. The spectrum further steepens to $\alpha \sim-1$ in the southernmost parts of the eastern plume (see Fig. 5). Near the peak of the eastern plume, we find $\alpha \sim-0.7$. Such a smooth variation in the spectral index away from the core is characteristic of WATs (see e.g., Hardcastle 1998).

\subsection{Interesting radio sources located near the WAT}

We also determined the spectral indices of the radio sources found within half a degree of the cD galaxy. The flux densities of the objects obtained from our 240 MHz map and from the FIRST (Becker et al. 1995) were used unless the object was extended. (in which case NVSS data by Condon et al. (1998) were used). Two interesting objects were found and we list their flux densities and spectral indices in Table 2. The first object (GMRT1) has an ultra-steep spectrum with $\alpha=-1.2$ and no optical counterpart is seen in the SDSS. The source is not resolved in the 


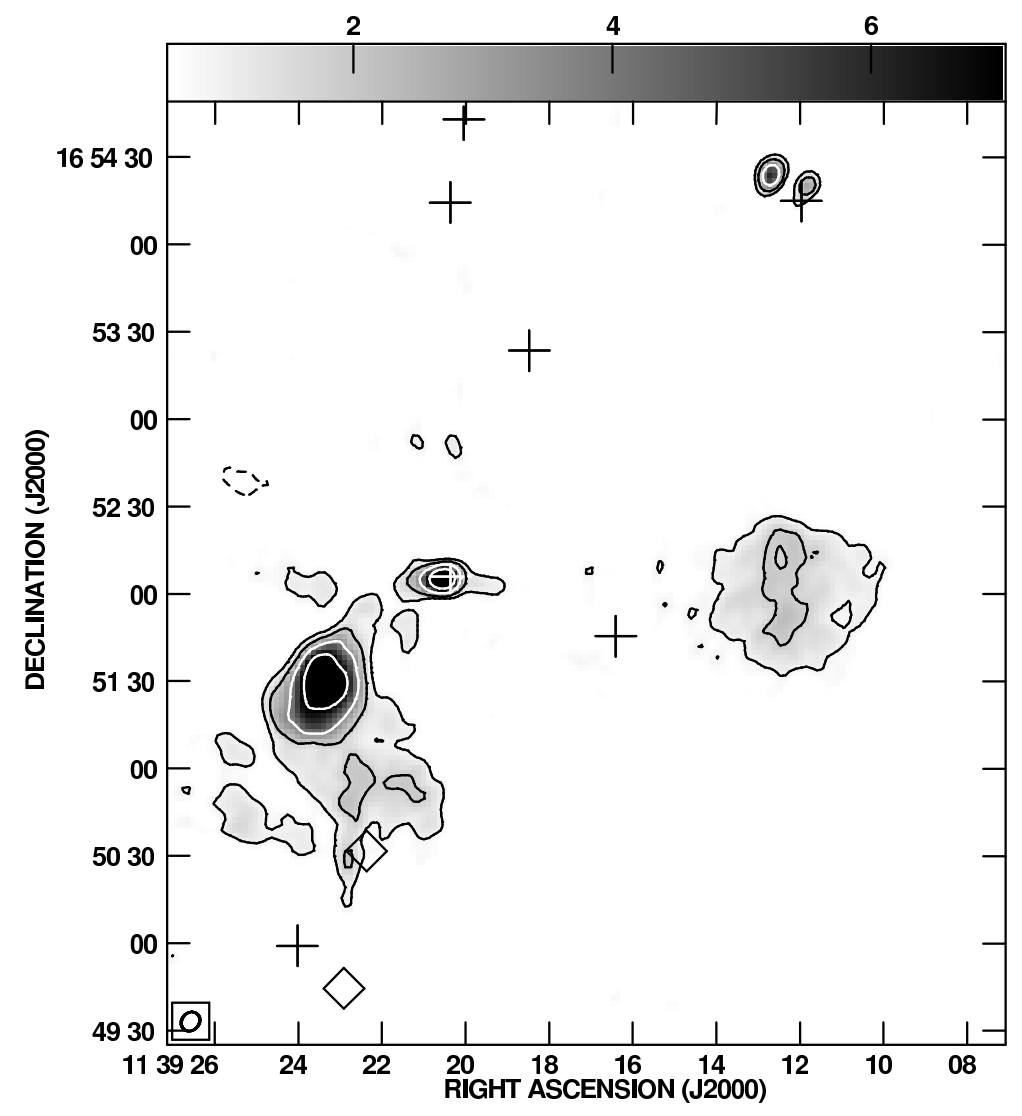

Figure 3. (a) The WAT associated with PGC 1519010 in $610 \mathrm{MHz}$ radio continuum imaged using the GMRT. This image was made using Briggs robust $=0$ and has an angular resolution of $7^{\prime \prime} \times 6^{\prime \prime}$ at a position angle of $-40.3^{\circ}$. The first contour is $0.9 \mathrm{mJy} / \mathrm{beam}$ and contours are subsequently plotted in multiples of 2 . Notice the C-shaped morphology of the radio structure and the clearly bent jet/plume in the east. The explanation of the symbols are as in Fig. 2b.

Table 2. Two interesting radio sources found within $15^{\prime}$ of the WAT radio galaxy. The 1.4 GHz flux density for GMRT1 is from FIRST and for GMRT2 is from NVSS data. Note that the radio position of GMRT1 and the position of the optical counterpart of GMRT2 are listed here. We use $S \propto \nu^{\alpha}$.

\begin{tabular}{c|c|c|c|c|c|c}
\multicolumn{1}{l}{ listed here. We use $S \propto \nu^{\alpha}$} & $\begin{array}{c}\alpha_{2000} \\
\text { hh mm ss.s }\end{array}$ & $\begin{array}{c}\delta_{2000} \\
\text { dd }, ., .\end{array}$ & $\begin{array}{c}\mathrm{S}_{1420 M H z} \\
\text { mJy }\end{array}$ & $\begin{array}{c}\mathrm{S}_{610 M H z} \\
\text { mJy }\end{array}$ & $\begin{array}{c}\mathrm{S}_{240 M H z} \\
\text { mJy }\end{array}$ & $\alpha_{1400}^{240}$ \\
\hline GMRT1 & 113927.3 & 164550.0 & 68.7 & 136 & 578 & -1.2 \\
GMRT2 & 113823.1 & 165150.1 & 24.8 & 34.5 & 107.3 & -0.82 \\
\hline
\end{tabular}




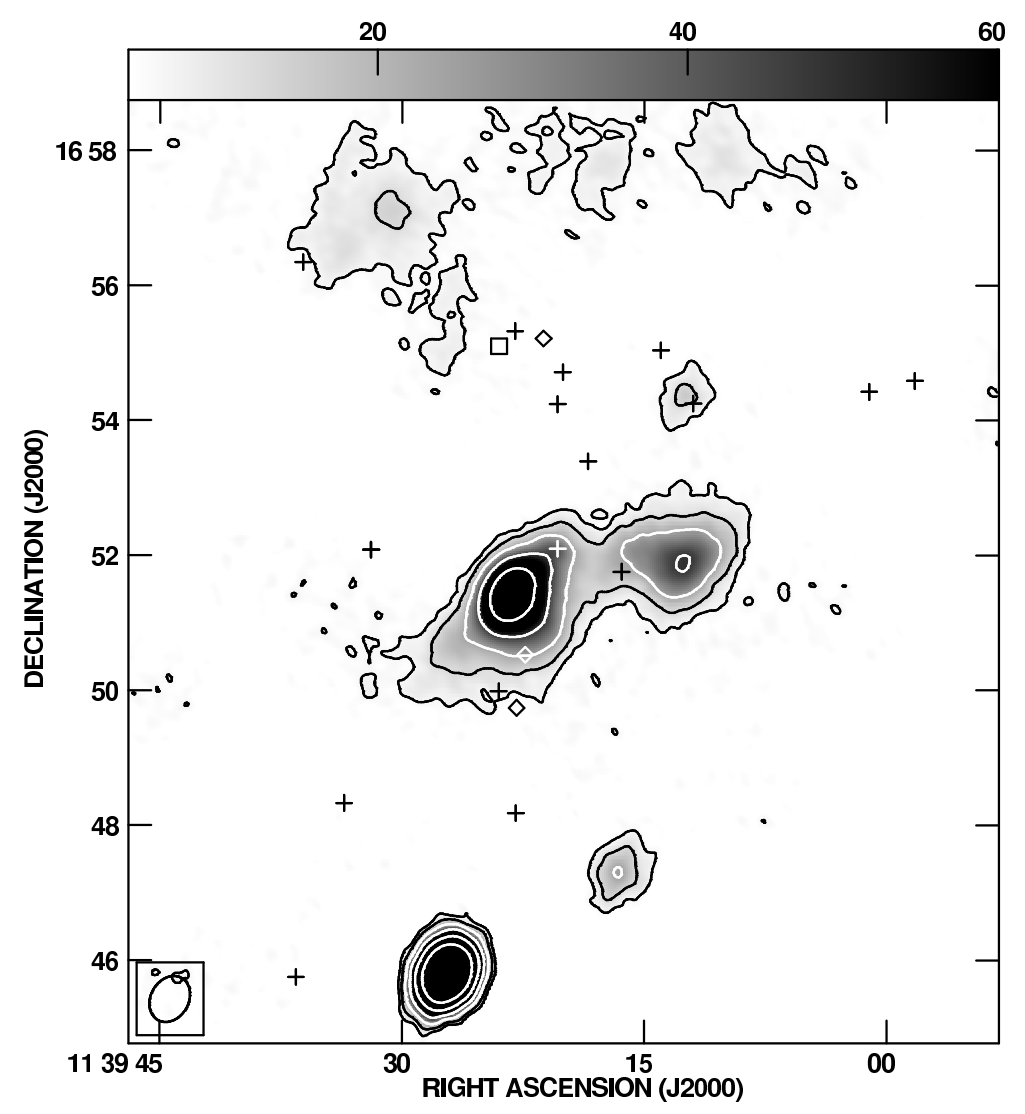

Figure 3. (b) The WAT associated with PGC 1519010 in $240 \mathrm{MHz}$ radio continuum imaged using the GMRT. Notice the double-lobed structure around the core of the galaxy. The 240 $\mathrm{MHz}$ image was made using natural weighting with Briggs Robust=5 and has an angular resolution of about $43 " \times 34^{\prime \prime}$ at a position angle of $-29.4^{\circ}$. The lowest plotted contour is $6 \mathrm{mJy} / \mathrm{beam}$ and the contours then increase in multiples of 2 . The explanation of the symbols are as in Fig. $2 b$.

highest resolution maps which translates to an upper limit on its angular extent of $1.8^{\prime \prime} \times 0.6^{\prime \prime}$ at a position angle of $80^{\circ}$ obtained from the FIRST. The nearest galaxy listed by SDSS is $15^{\prime \prime}$ away from GMRT1. This strong ultra-steep spectrum object is located $\sim 3^{\prime}$ to the south of the eastern lobe of the WAT (Fig. 2b, 3b, 7).

The second object (GMRT2) listed in Table 2 lies to the west of the $\mathrm{cD}$ and is an extended source. SDSS lists five galaxies within $0.5^{\prime}$ of the radio source which are shown by crosses in Fig. 6. All are faint objects without reliable redshift estimates. Our $610 \mathrm{MHz}$ image (Fig. 6) has picked up faint extended emission which shows that the source is, in fact, a highly asymmetric double of total angular size $\sim 80^{\prime \prime}$. The likely counterpart is marked with a star in Fig. 6. If this source lies at the distance of the WAT, then its linear size would be about $180 \mathrm{kpc}$. However 


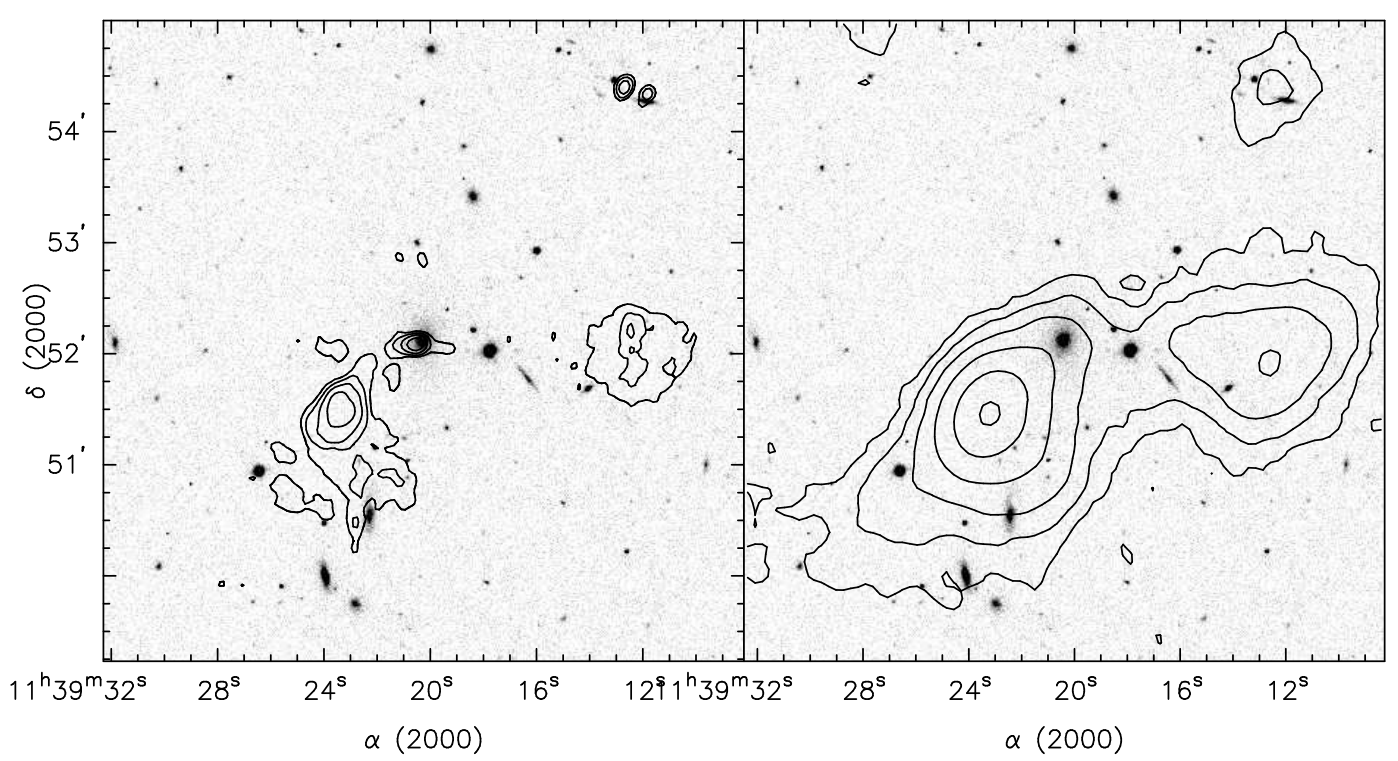

Figure 4. The WAT radio source associated with PGC 1519010 in the $610 \mathrm{MHz}$ (left) and $240 \mathrm{MHz}$ (right) radio continuum superposed on the SDSS R band image plotted on the same angular scale. The angular resolution of the $610 \mathrm{MHz}$ image is $7^{\prime \prime} \times 6^{\prime \prime}$ with a position angle of $-40.3^{\circ}$ whereas it is $43^{\prime \prime} \times 34^{\prime \prime}$ at a position angle of $-29.4^{\circ}$ at $240 \mathrm{MHz}$. The bright optical object between the western jet and plume is classified as a star in the SDSS.

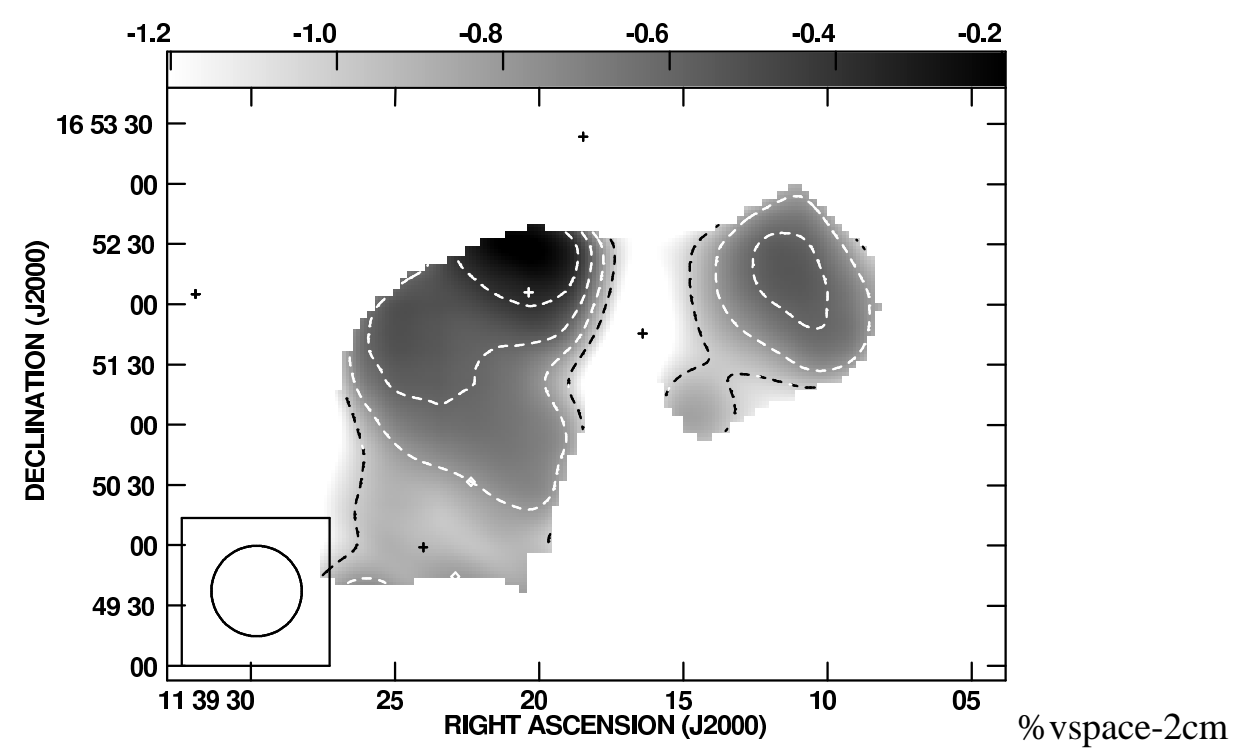

Figure 5. The low resolution ( $\left.45^{\prime \prime}\right)$ spectral index distribution between 240 and $1.4 \mathrm{GHz}$. Note the gradual steepening of the spectrum along the jet and plume in the east with increasing separation from the core. 


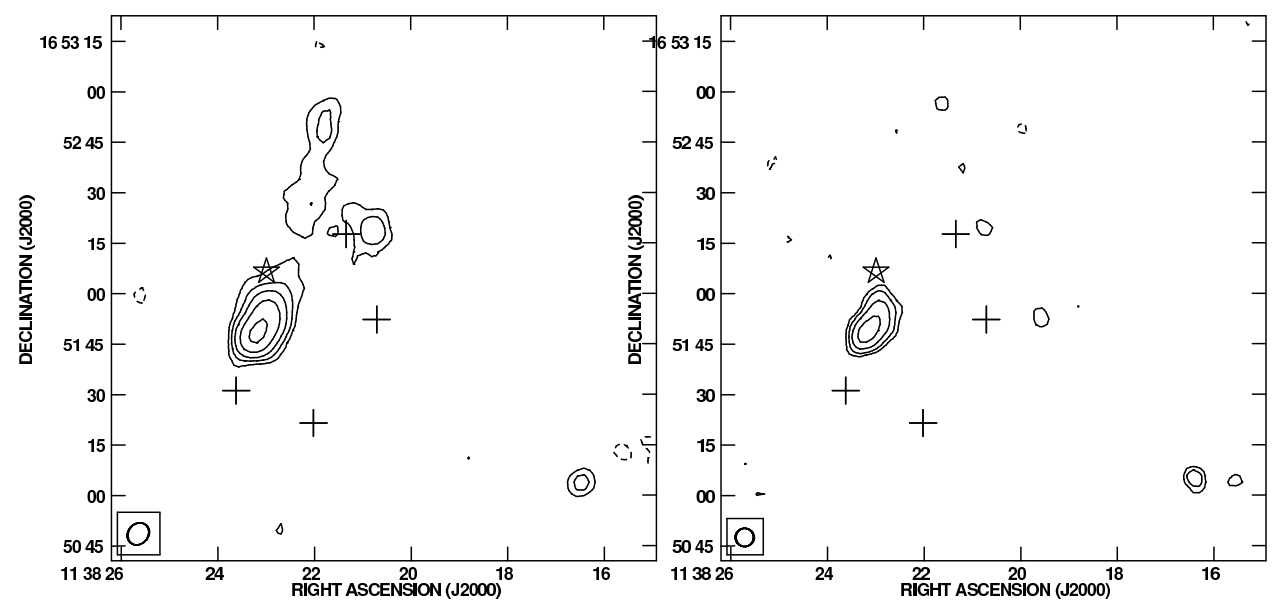

Figure 6. The radio source GMRT2 located to the west of the WAT (Table 2). The 610 MHz GMRT image is shown on the left and the $1.4 \mathrm{GHz}$ image from FIRST is shown on the right. The lowest contour at $610 \mathrm{MHz}$ is $0.6 \mathrm{mJy} / \mathrm{beam}$ and at $1.4 \mathrm{GHz}$ is $0.45 \mathrm{mJy} / \mathrm{beam}$ and subsequently increase in multiples of 2 . Notice the extended faint emission detected at $610 \mathrm{MHz}$ which is not seen in the FIRST map. Based on this detection, the star marks the position of the likely optical counterpart of the asymmetric double radio source whereas the plus signs (+) mark the positions of the remaining SDSS galaxies found within 30" of the radio peak.

since the host galaxy is faint, this object is likely to be more distant and therefore physically large.

\section{Discussion}

\subsection{A new WAT and its associated cluster}

The galaxy PGC 1519010, as inferred here from its optical morphology and the association with a powerful WAT, is a cD galaxy (Table 1).

As mentioned above, the most striking and peculiar morphological feature of this WAT is the contrast between the jet/plume bending on the opposite sides of the core, such that the bending is found exclusively on the eastern side. In order to investigate this further, we have inspected the SDSS database. Gal et al. (2003), using the SDSS have identified a cluster (NSC 113924+165506) centred $\sim 3^{\prime}$ north-east of PGC 1519010 (see Fig.3, the square symbol) and having a photometric redshift of 0.1365 , which is close to the value estimated for the WAT. To probe this further, we show in Fig. 7 the distribution of the galaxies listed in SDSS DR6. For the cD progenitor of the WAT, SDSS J113920.37+165206, the SDSS provides three estimates of $z_{\text {phot }}=0.138,0.112$ and 0.104 . These are in reasonable agreement with the afore-mentioned value for the cluster $\left(z_{p h o t}=0.1365\right.$, Gal et. al. 2003). In order to trace the optical field near this region we searched the SDSS 
for galaxies in the adjacent area and found three galaxies within $\sim 4^{\prime}$ of the cD galaxy PGC 1519010 (WAT), having spectroscopic redshifts between 0.105 and 0.107. These include U1-8 (U1-8, O'Neil et al. 1997), a LSB galaxy lying about $2^{\prime}$ south of the cD, for which Bergmann et al. (2003) have estimated a spectroscopic redshift of 0.10715 (photometric redshifts given in the SDSS catalogue are 0.141 , 0.095 and 0.091 ). The second galaxy, SDSS J113921.25+165512.8 is close to U1-8 and SDSS database gives a spectroscopic redshift of 0.105 whereas the photometric redshifts listed there are $0.121,0.118$ and 0.108 . The third galaxy lies about $3.5^{\prime}$ north of the $\mathrm{cD}$ and SDSS gives a spectroscopic redshift of 0.105 whereas the photometric redshifts are quoted there to be 0.061, 0.094 and 0.0.095. All these spectroscopic redshift strongly suggest that the true redshift of the $\mathrm{cD}$ (WAT) is close to 0.106 , which would also be in reasonable accord with its photometric redshifts mentioned above. Taking $z=0.106$ for the $\mathrm{cD}$, the radio luminosities of the

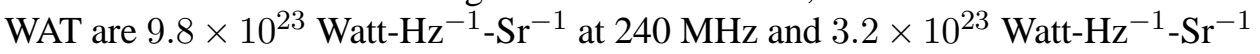
at $1.4 \mathrm{GHz}$. These values lie in the region of the FR I/II transition and are thus characteristic of WATs. The distances from the core at which the jet flaring occurs are about $90 \mathrm{kpc}$ and $230 \mathrm{kpc}$, for the eastern and western jets, respectively.

To probe the large scale environment around the cD, we examined the SDSS DR6 data over a $1^{\circ}$ circular region centred at the $\mathrm{cD}$. In particular, we searched for the galaxies for which a physical association with the $\mathrm{cD}$ is very probable. The criterion we employed was that the photometric redshifts of the optical objects should be between 0.08 and 0.41 . A relatively wide range had to be admitted since the spectroscopic redshifts are available for only a few galaxies in the region. We found that the range roughly represents the typical scatter among the three SDSS estimates of the photometric redshifts for the objects in this region.

The 37 objects that we selected from the above criterion include only five galaxies with known spectroscopic redshifts. Nonetheless, they can be expected to manifest the gross features of the galaxy clustering associated with the the $\mathrm{cD}$ (WAT) (see Fig. 7). Note also that in the soft X-ray band the ROSAT (Voges et al. 1999 and references therein) database shows a diffuse source of about $0.5^{\circ}$ diameter, covering this region. The detection of hot gas is consistent with the proposed galaxy clustering scenario. As mentioned above, Gal et al. (2003) have reported a galaxy cluster with Abell richness 0 which is close in both redshift and direction to the galaxy group we identify here to be associated with the WAT.

\subsection{Origin of the WAT}

Perhaps the most remarkable feature emerging from the galaxy distribution (Fig. 7) is a nearly $0.5 \mathrm{Mpc}$ long chain of galaxies stretching from the cD roughly northsouth towards the cluster centre defined by Gal et al. (2003) and possibly extending also to the south of the $\mathrm{cD}$. It is along this filament that galaxies are likely to have approached the $\mathrm{cD}$ prior to merging with it. This galaxy merger scenario is further supported by the shape of the stellar halo of the $\mathrm{cD}$ which too is extended roughly

north-south. Thus it appears plausible that a bulk motion of the intergalactic gas has 
been occuring along this galaxy filament. This circumstance may provide potentially interesting clues about the mechanism of the jet disruption and the jet/plume bending in this WAT.

Following upon the early attempts to understand the nature of WATs (e.g., Eilek et al. 1984, Leahy 1984, O'Dea \& Owen 1985), a number of physical scenarios have been put forward to explain this rare type of phenomenon (Sect. 1). In some of the models, the jet disruption occurs as the jet crosses the ISM of the host galaxy into the ICM, and is thereby subject to either a steep density gradient (Sakelliou \& Merrifield 1999), or a side-way ram pressure ("crosswind" arising from bulk motion of the ICM, see Loken et al. 1995). In another scenario, the plumes form as the radio lobes of moderately powerful twin-jets are driven outwards due to buoyancy forces (Hardcastle 1999). The latter model is motivated by the observations that the jet in some WATs continue well into the plume (e.g., Hardcastle 1999; Hardcastle \& Sakelliou 2004).

Despite the modest sensitivity of our radio maps, the present WAT offers some insight and a broad check on some of the proposed models. This is because of the morphological contrast observed between its two jet/plume structures, eventhough the kinetic powers of the two jets are expected to be similar. Firstly, in this WAT a correlation is clearly seen between the bending properties of the jet and the resulting plume. As seen from Figs. 2, and 4, the western jet must be propagating straight for about $230 \mathrm{kpc}$ before flaring and the resulting radio plume likewise shows no sign of bending. A contrasting pattern is seen on the eastern side of the core, where the collimated jet undergoes a steady bending until its disruption and thereafter the resulting plume too exhibits a sharp bend consistent with the bent trajectory of the eastern jet. From this correlated behaviour it appears that a viable mechanism for jet disruption in WATs should be able to bend both the plume and the associated jet. This requirement casts some doubt on the mechanism which seeks to explain the jet disruption in terms of collision with a dense gas cloud (Sect. 1). Indeed, the observed alignment of the bent eastern plume with the chain of galaxies (see Fig. 7) would seem to be basically consistent with the ram pressure scenario involving bulk relative motion between the ICM and the radio galaxy. The observed radio structure would also imply that the crosswind is in the NE-SW direction with the plume finally aligning with it (see Fig. 3a). However, were the ram pressure of the ICM crosswind effective only after the jet crosses the ISM/ICM interface, it would be hard to explain the steady bending of the preceding collimated portion of the jet. We may recall that a similar difficulty for the basic crosswind scenario (e.g., Loken et al. 1995) has been noticed in the case of the WAT 0647+693 where the $50 \mathrm{kpc}$ long collimated western jet exhibits a steady bending before its disruption (Hardcastle \& Sakelliou 2004). It appears, therefore, that the effect of the ICM crosswind moving along the galaxy filament which may eventually cause the sharp bending of the plume and its alignment along the filament also acts on the progenitor jet before its flaring. In fact, for the head-tail radio galaxy NGC 1265, Jones \& Owen (1979) have proposed that a pressure gradient set up in the ISM due to the motion of the galaxy against ICM can bend its jet within the ISM, provided their Mach number 
is not too large. But, even if such a mechanism is viable for the present case, it will be required to explain the near absence of bending of the western jet and its radio plume (Fig. 3a \& 4). It would seem ad hoc to explain the contrasting pattern on the two sides by postulating a much larger kinetic power for the western jet. A conceivable alternative would be to postulate the existence of inhomogeneities in the ICM on $100 \mathrm{kpc}$ scale, but here too the inhomogeneity will have to be positioned, so as to operate on just one side of the nucleus. Nonetheless, this possibility needs to be investigated further. As a first step, it would be useful to make a deeper radio image of this WAT, in order to look for any bent faint extensions of the western plume and to trace the trajectories of the two jets more clearly. Secondly, this object is an excellent target for multi-object spectroscopy needed for a better delineation of the galaxy distribution around the $\mathrm{cD}$. Finally, targeted X-ray imaging of this region is needed to establish the morphological details of the ROSAT detected diffuse $\mathrm{X}$-ray source, so that the suspected filamentary gravitational potential well can be properly traced.

\section{Summary}

In this paper, we report the GMRT detection of a new wide-angle-tail radio source associated PGC 1519010 which we find to be a cD galaxy. These observations at 610 and $240 \mathrm{MHz}$ were originally aimed at studying the low surface brightness galaxy located 16' to the north. The power of the radio source at $240 \mathrm{MHz}$ is $\sim 10^{24}$ Watt- $\mathrm{Hz}^{-1}-\mathrm{Sr}^{-1}$ which is close to the FRI/FRII break. Using the SDSS DR6 database, we have identified a cluster of 37 galaxies likely to be associated with the WAT. Further the ROSAT All Sky Survey shows faint diffuse extended Xray emission in the same region which indicates the presence of a hot intracluster medium. Using the spectroscopic redshifts available for a few galaxies close to the $\mathrm{cD}$, we suggest that the redshift of the group/cluster of galaxies associated with the WAT is 0.106 . Moreover, we note that the clustering of member galaxies close to the WAT indicates a filament along which galaxies have been merging with the cD. This scenario also finds support in the observed NS extension of the stellar halo of the cD galaxy.

Our radio observations have highlighted the peculiar morphology of this WAT radio source. The source shows constrasting morphology on the two sides of the core: the eastern jet emerges from the core and undergoes a gradual bending before being disrupted to form the plume which itself continues to bend in the same direction. On the western side, the jet emerging from the core appears to continue straight for about $230 \mathrm{kpc}$, before being disrupted to form the faint plume which too shows no bent extension. The very different behaviour on the two sides suggests that the mechanism responsible for the plume bending is also causing the jet to bend. Several mechanisms for jet bending have been put forward and separate mechanisms have also been invoked to explain the jet and plume bending (Sect. 1). However, from our present results for the WAT associated with PGC 1519010, the 


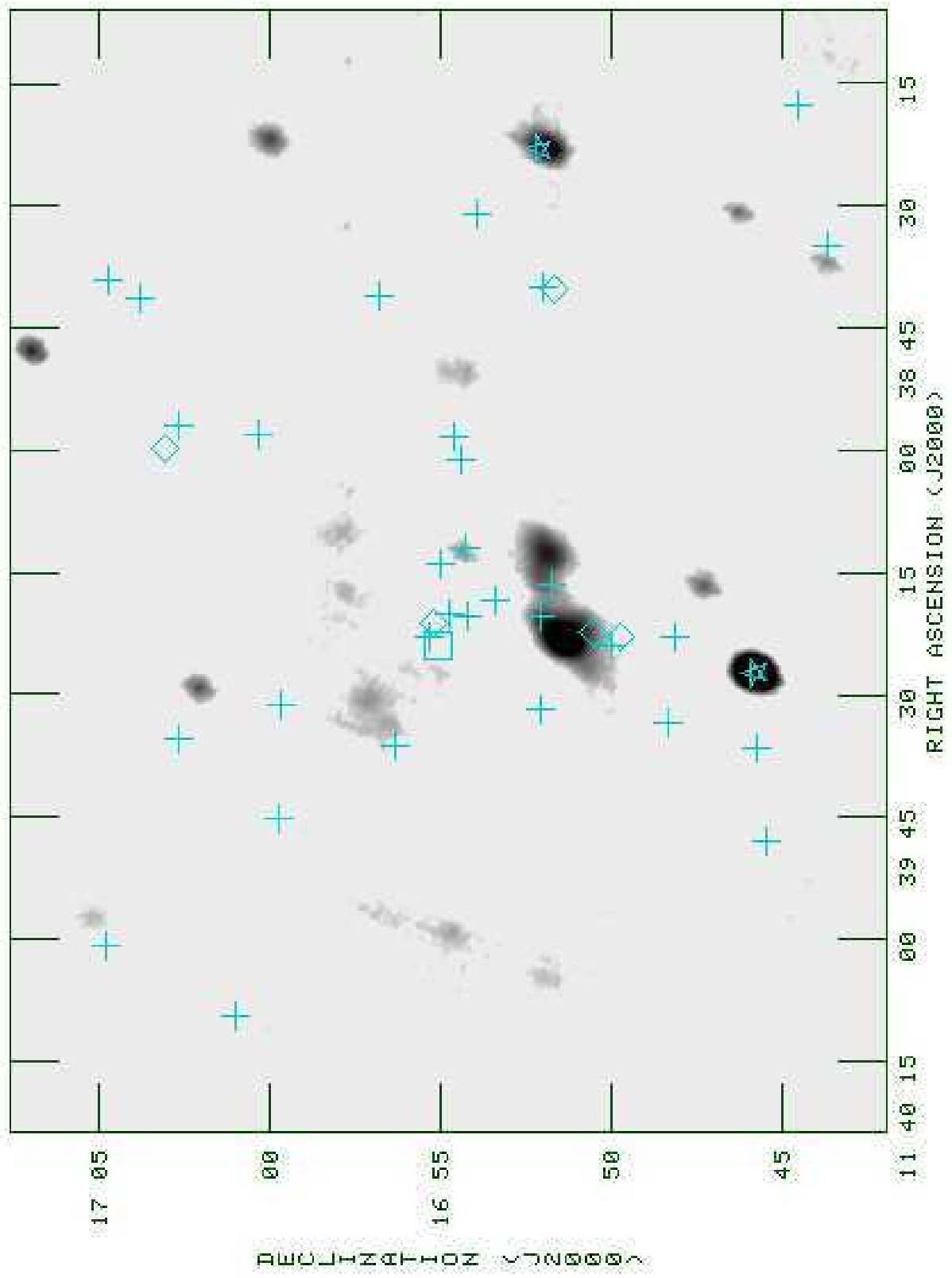

Figure 7. $240 \mathrm{MHz}$ GMRT image of the WAT made with robust weighting=5 is shown. The positions of 37 galaxies which are likely to be associated with the WAT are marked in the figure. Five of them with known spectrocopic redshift are marked with diamonds. The remaining 32 member galaxies are marked by plus (+) sign. The square marks the position of the cluster centre NSC $113924+165506(z=0.1365)$. as given by Gal et al. (2003). The cluster is about 20' $(\sim 2.5 \mathrm{Mpc})$ in size. Note the almost NS chain of galaxies extending southwards from the cluster centre towards the $\mathrm{cD}$. The two interesting radio sources GMRT1 and GMRT2 (Table 2) are marked by stars. 
similar bending shown by the jet and the plume on the east of the core suggests that the same bending mechanism is operating on both components.

Several theories have tried to explain the WAT radio morphology. From our data, we favour the crosswind mechanism (Loken et al. 1995) wherein the jet runs into the crosswind due to the merging of clusters and which, in turn, exerts ram pressure on the jet/plume, causing it to bend. We note that the eastern jet continues for about $90 \mathrm{kpc}$ before being disrupted into a plume. Around this distance, the jet would have crossed the hot ISM of the host elliptical galaxy. and would encounter the lower density ICM, causing it to flare. The direction of the bending of the jet/plume agrees with the filament of galaxies and the extension of the cD stellar halo. Diffuse X-ray emission too is extended roughly in the NS direction, lending further support to the crosswind mechanism. However, explaining the unaffected/unbent western jet/plume is difficult unless inhomogeneities in the ICM are invoked. More sensitive and high angular resolution observations are thus required to obtain a better understanding of this interesting system.

We also report the detection of an ultra-steep spectrum source $(\alpha=-1.2)$ to the south of the eastern plume, which does not have an optical counterpart in SDSS. Another interesting source we have found is a highly asymmetric radio double source located to the west of the WAT.

\section{Acknowledgements}

We thank the staff of the GMRT who have made these observations possible. GMRT is run by the National Centre for Radio Astrophysics of the Tata Institute of Fundamental Research. This research has made use of the Sloan Digital Sky Survey (http://www.sdss.org) Data Release 6 (SDSS DR6), the Faint Images of Radio Sky at Twenty cms (FIRST) survey, the NRAO VLA Sky Survey (NVSS), NASA's Astrophysics Data System (ADS), ROSAT Data Archive (of the Max-Planck-Institut fr extraterrestrische Physik (MPE) at Garching, Germany), NASA/IPAC Extragalactic Database (NED) (which is operated by the Jet Propulsion Laboratory, California Institute of Technology, under contract with the National Aeronautics and Space Administration), and Hyperleda (http://leda.univ-lyon1.fr).

\section{References}

Ananthakrishnan, S. \& Rao, A. P., 2002, Multicolour Universe, Ed. Manchanda, R. \& Paul, B., p233.

Becker, R. H., White, R. L., Helfand, D. J., 1995, Astrophy. J., 450, 559.

Bergmann, M. P., Jorgensen, I., Hill, G. J., 2003, Astron. J., 125, 116

Blanton, E., Gregg, M. D., Helfand, D. J., Becker, R. H., White, R. L., 2000, Astrophy. J., 531, 118

Blanton, E., Gregg, M. D., Helfand, D. J., Becker, R. H., Leighly, K. M., 2001, Astron. J., 121, 2915 
Blanton, E., Gregg, M. D., Helfand, D. J., Becker, R. H., White, R. L., 2003, Astron. J., 125, 1635

Briggs, D., High Fidelity Deconvolution of Moderately Resolved Sources, PhD thesis, 1995

Burns, J.O., White, R. A., Hough, D. H., 1981, Astron. J., 86, 1

Burns, J. O., Rhee, G., Owen, F. N., Pinkney, J., 1994, Astrophy. J., 423, 94

Condon, J. J., Cotton, W. D., Greisen, E. W., Yin, Q. F., Perley, R. A., Taylor, G. B., Broderick, J. J., 1998, Astron. J., 115, 1693

Das, M., Kantharia, N. G., Ramya, S., Prabhu, T. P., McGaugh, S. S., Vogel, S. N., 2007, Mon. Not. R. Astron. Soc., 379, 11

Das, M., McGaugh, S. S, Kantharia, N. G., Vogel, S. N., 2008 in Dark Galaxies and Lost Baryons, Proceedings of the IAU Symposium, 244, 352.

Doe, S. M., Ledlow, M. J., Burns J. O., White, R. A., 1995, Astron. J., 110, 46.

Eilek, J., Burns, J. O., O’Dea, C. P., Owen, F. N., 1984, Astrophy. J, 278, 37.

Fanaroff, B. L., Riley, J. M., 1974, Mon. Not. R. Astron. Soc., 167, 31

Gal, R. R., De Carvalho, R. R., Lopes, P. A. A., Djorgovski, S. G., Brunner, R. J., Mahabal, A., Odewahn, S. C., 2003, Astron. J., 125, 2064

Gopal-Krishna, Wiita, P. J., 1987, Mon. Not. R. Astron. Soc., 226, 531

Gomez, P. L., Ledlow, M. J., Burns, J. O., Pinkey, J., Hill, J. M., 1997, Astron. J., 114, 1711

Hardcastle, M. J., 1998, Mon. Not. R. Astron. Soc., 298, 569

Hardcastle, M. J., 1999, Astron. \& Astrophy., 349, 341

Hardcastle, M. J., Sakelliou, I., 2004, Mon. Not. R. Astron. Soc., 349, 560

Hardcastle, M. J., Sakelliou, I., Worrall, D. M., 2005, Mon. Not. R. Astron. Soc., 359, 1007

Higgins, S. W., O’Brien, T. J., Dunlop, J. S., 1999, Mon. Not. R. Astron. Soc., 209, 273

Hooda, J. S., Wiita, P. J., 1996, Astrophy. J, 470, 21

Jetha, N., Hardcastle, M., Sakelliou, I., 2006, Mon. Not. R. Astron. Soc., 368, 609

Jones, T. W., Owen, F. N., 1979, Astrophy. J, 234, 818

King, I., 1962, Astron. J., 67, 471

King, I., 1966, Astron. J., 71, 276

Kormendy, J., 1977, Astrophy. J, 218, 333

Leahy, J. P., 1984, Mon. Not. R. Astron. Soc., 208, 323

Leahy, J. P., 1993, Jets in Extragalactic Radio Sources, Proceedings of a Workshop Held at Ringberg Castle, Tegernsee, FRG, September 22-28, 1991. Ed: H.-J. Rser and K. Meisenheimer. Springer-Verlag Berlin Heidelberg New York. Also Lecture Notes in Physics, volume 421, 1993, p.1

Loken, C., Roettiger, K., Burns, J. O., Normal, M., 1995, Astrophy. J, 445, 80

O’ Neil, K., Bothun, G. D., Cornell, M. E., 1997, Astron. J., 113, 1212

O’ Dea, C. P., Owen, F. N., 1985, Astron. J., 90, 927

O’Donoghue, Aileen, A., Owen, F. N., Eilek, J. A., 1990 Astrophy.J. Supple. Ser., 72, 75

O’Donoghue, Aileen, A., Eilek, J. A., Owen, F. N., 1993 Astrophy. J, 408, 428

Owen, F. N., Rudnick, L., Astrophy. J, 205, L1

Paturel, G., Petit, C., Prugniel, P., Theureau, G., Rousseau, J., Brouty, M., Dubois, P., Cambrésy, L. 2003, Astron. \& Astrophy., 412, 45

Sakelliou, I., Merrifield, M. R., 1999, Mon. Not. R. Astron. Soc., 305, 417

Swarup, G., Ananthakrishnan, S., Kapahi, V. K., et al. 1991, Current Science, 60, 95.

Voges, W., et al., 1999, Astron. \& Astrophy., 349, 389 